In Cooperation with the National Park Service and the U.S. Department of Agriculture Forest Service

\title{
Rocky Mountain Snowpack Chemistry at Selected Sites, 2004
}

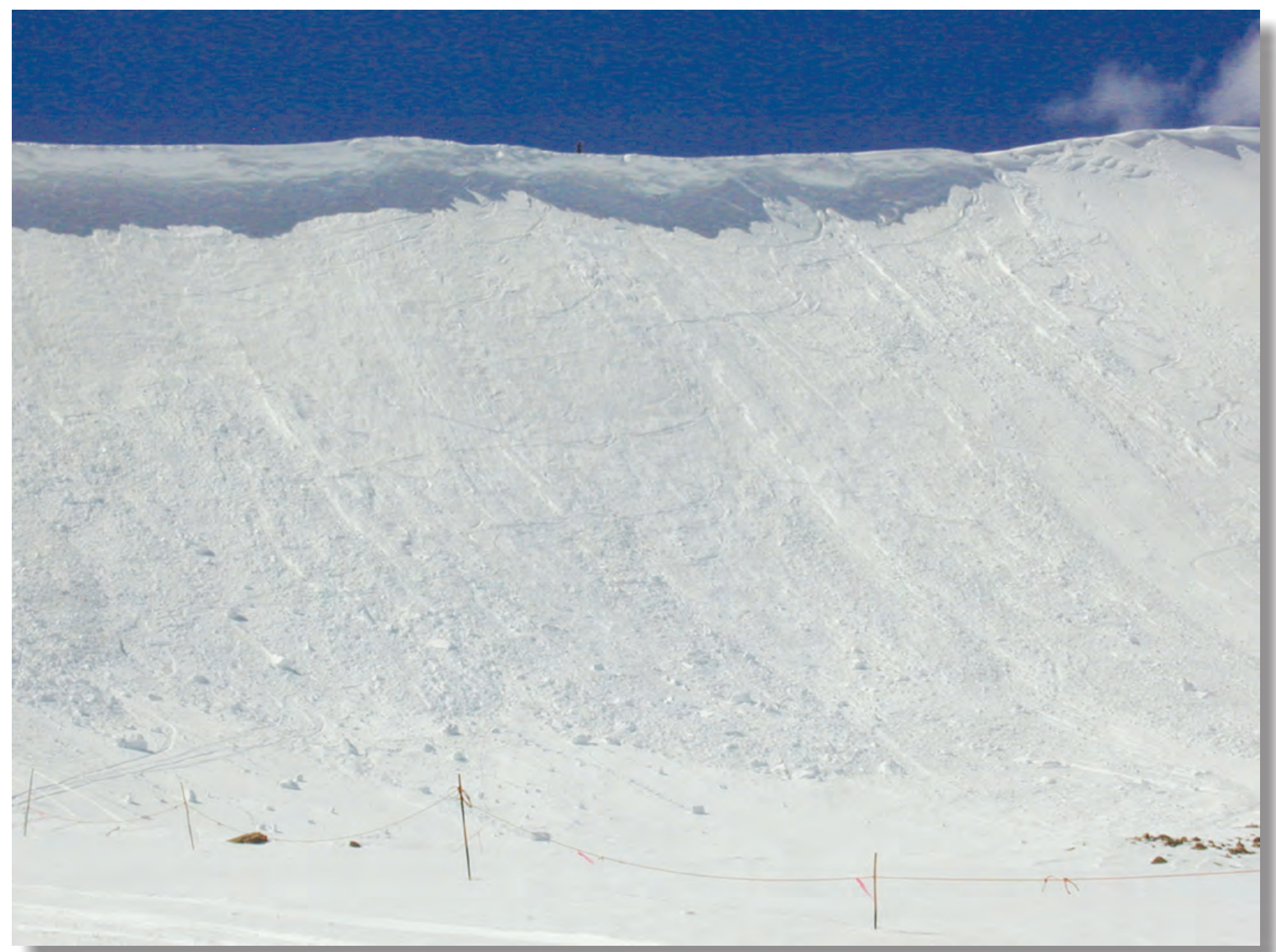

Open-File Report 2007-1045 


\section{Rocky Mountain Snowpack Chemistry at Selected Sites, 2004}

By George P. Ingersoll, M. Alisa Mast, Leora Nanus, Heather H. Handran, David J. Manthorne, and Douglas M. Hultstrand

In Cooperation with the National Park Service and the

U.S. Department of Agriculture, Forest Service

Open-File Report 2007-1045 


\title{
U.S. Department of the Interior DIRK KEMPTHORNE, Secretary
}

\author{
U.S. Geological Survey \\ Mark D. Myers, Director
}

U.S. Geological Survey, Reston, Virginia 2007

For product and ordering information:

World Wide Web: http://www.usgs.gov/pubprod

Telephone: 1-888-ASK-USGS

For more information on the USGS - the Federal source for science about the Earth, its natural and living resources, natural hazards, and the environment: World Wide Web: http://www.usgs.gov

Telephone: 1-888-ASK-USGS

Any use of trade, firm, or product names is for descriptive purposes only and does not imply endorsement by the U.S. Government

Although this report is in the public domain, permission must be secured from the individual copyright owners to reproduce any copyrighted material contained within this report.

Suggested citation:

Ingersoll, George P., Mast, M. Alisa, Nanus, Leora, Handran, Heather H., Manthorne, David J., and Hultstrand,

Douglas M., 2007, Rocky Mountain snowpack chemistry at selected sites, 2004: U.S. Geological Survey

Open-File Report 2007-1045, 15 p. 


\section{Contents}

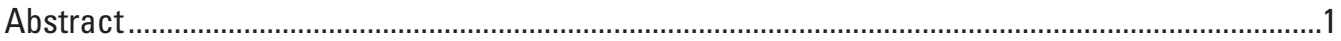

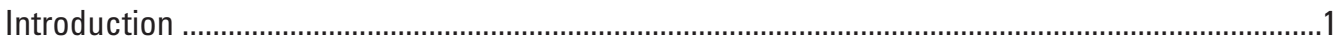

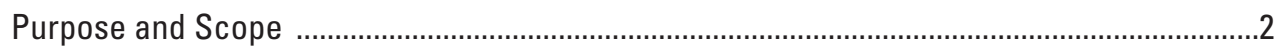

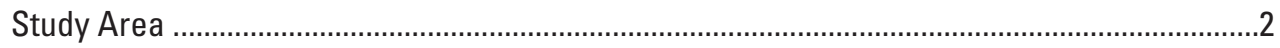

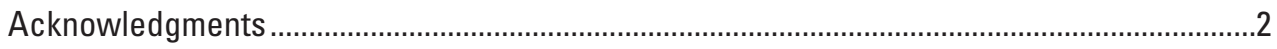

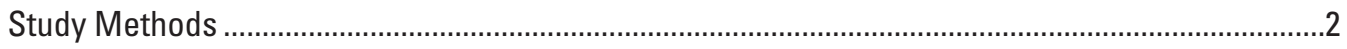

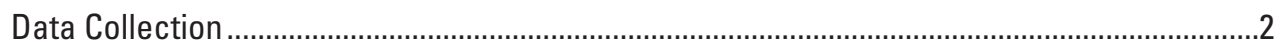

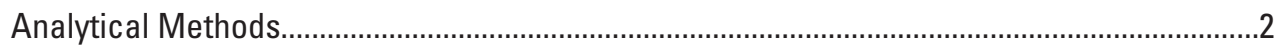

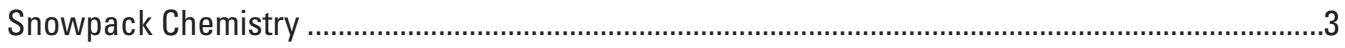

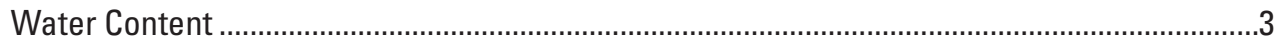

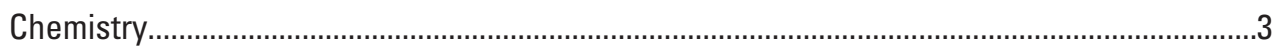

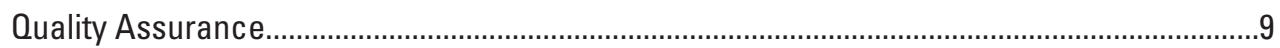

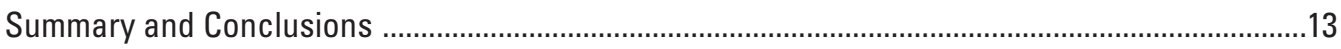

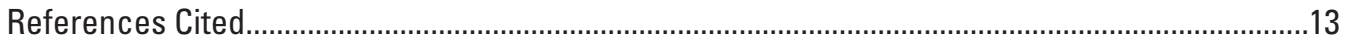

\section{Figures}

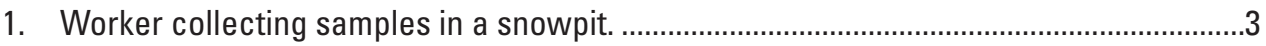

2-6. Maps showing:

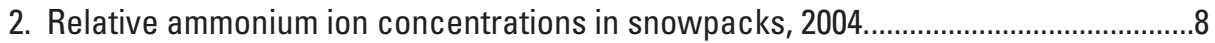

3. Relative nitrate ion concentrations in snowpacks, 2004...............................................

4. Relative sulfate ion concentrations in snowpacks, 2004.............................................10

5. Relative total mercury concentrations in snowpacks, 2004.......................................12

6. Relative stable sulfur isotope ratios in snowpacks, 2004. ...........................................12

\section{Tables}

1. Snow depth and snow-water equivalent; $A N C$ and laboratory $\mathrm{pH}$; average concentrations of major ions, dissolved organic carbon, mercury, and sulfur-34 ; and ionic charge balances from analyses of the 2004 snowpack .................4

2. Quality assurance field and laboratory blank results for ammonium, sulfate, nitrate (microequivalents per liter)....

3. Quality assurance replicate sample results for ammonium, sulfate, nitrate, mercury, and sulfur-34. 


\section{Conversion Factors}

\section{SI to Inch/Pound}

\begin{tabular}{lll}
\hline \multicolumn{1}{c}{ Multiply } & \multicolumn{1}{c}{ By } & \multicolumn{1}{c}{ To obtain } \\
\hline \multicolumn{1}{c}{ Length } \\
\hline centimeter $(\mathrm{cm})$ & 0.3937 & inch \\
meter $(\mathrm{m})$ & 3.281 & foot \\
kilometer $(\mathrm{km})$ & 0.6214 & mile \\
\hline \multicolumn{2}{c}{ Volume } & ounce, fluid \\
\hline liter $(\mathrm{L})$ & 33.82 & \\
\hline \multicolumn{2}{c}{ Mass } \\
Nanogram $(\mathrm{ng})$ & $3.53 \times 10^{-11}$ & ounce \\
\hline
\end{tabular}

To convert microequivalents per liter ( $\mu \mathrm{eq} / \mathrm{L}$ ) to milligrams per liter for major ions, divide microequivalents by factors indicated for each ion:

\begin{tabular}{lll}
\hline To obtain milligrams per liter & for & divide by \\
\hline $\mathrm{H}^{+}$ & 1000 \\
$\mathrm{Ca}^{2+}$ & 49.90 \\
$\mathrm{Mg}^{2+}$ & 82.26 \\
$\mathrm{~K}^{+}$ & 25.57 \\
$\mathrm{Na}^{+}$ & 43.50 \\
$\mathrm{NH}_{4}^{+}$ & 55.44 \\
$\mathrm{SO}_{4}^{-}$ & 20.83 \\
$\mathrm{NO}^{-}$ & 16.13 \\
$\mathrm{Cl}^{-}$ & 28.21 \\
\hline
\end{tabular}

Temperature in degrees Celsius $\left({ }^{\circ} \mathrm{C}\right)$ may be converted to degrees Fahrenheit $\left({ }^{\circ} \mathrm{F}\right)$ as follows:

$$
{ }^{\circ} \mathrm{F}=\left(1.8 x^{\circ} \mathrm{C}\right)+32
$$

Vertical coordinate information is referenced to the North American Vertical Datum of 1988 (NAVD 88).

Horizontal coordinate information is referenced to the North American Datum of 1983 (NAD 83).

Altitude, as used in this report, refers to distance above the vertical datum. 


\title{
Rocky Mountain Snowpack Chemistry at Selected Sites, 2004
}

\author{
By George P. Ingersoll, M. Alisa Mast, Leora Nanus, Heather H. Handran, David J. Manthorne, and Douglas \\ M. Hultstrand
}

\section{Abstract}

During spring 2004, the U.S. Geological Survey in cooperation with the National Park Service and the U.S. Department of Agriculture, Forest Service collected and analyzed snowpack samples for 65 sites in the Rocky Mountain region from New Mexico to Montana. Snowpacks were sampled from late February through early April and generally had well-below-average- to near-average snow-water equivalent. Regionally, on April 1, snow-water equivalent ranged from 50 to 89 percent.

At most regional sites monitored during 1993-2004, snowpack ammonium, nitrate, and sulfate concentrations for 2004 were lower than the 12-year averages. Snowpack ammonium concentrations in the region were lower than average concentrations for the period at 61 percent of sites in the region, but showed a new pattern compared to previous years with three of the four highest 2004 concentrations observed in northern Colorado. Nitrate concentrations in 2004 were lower than the 12-year average for the year at 53 percent of regional sites, and typically occurred at sites in Wyoming, Idaho, and Montana where powerplants and large industrial areas were limited. A regional decrease in sulfate concentrations across most of the Rocky Mountains (with concentrations lower than the 12-year average at 84 percent of snowpack sites) was consistent with other monitoring of atmospheric deposition in the Western United States. Total mercury concentrations, although data are only available for the past 3 years, decreased slightly for the region as a whole in 2004 relative to 2003. Ratios of stable sulfur isotopes indicated a similar regional pattern as observed in recent years with sulfur-34 $\left(\delta^{34} \mathrm{~S}\right)$ values generally increasing northward from northern New Mexico and southern Colorado to northern Colorado, Wyoming, Idaho, and Montana.

\section{Introduction}

Snowfall that accumulates from October until March, April, or May provides about 50 to 70 percent of the annual precipitation in headwater basins of the Rocky Mountains (Western Regional Climate Center, 2004). As these snowpacks accumulate during the winter and spring, chemicals deposited from the atmosphere are stored until snowmelt begins in spring. Because snowmelt supplies most of the freshwater in mountain lakes, streams, and wetlands, monitoring the water quality of snow is important to understanding the effects of atmospheric deposition to these systems.

In the Rocky Mountain region, population growth, water use, and energy development are increasingly affecting the quantity and quality of water resources at higher elevations (Fenn and others, 2003; Bureau of Land Management Wyoming, 2006; McGuire, 2006; U.S. Environmental Protection Agency, 2006a, b). Identifying changes in water quality and processes leading to degradation of water quality is important because alpine and subalpine environments in the region are sensitive to changes in chemical composition of the water. Thin alpine and subalpine soils and dilute water bodies in mountain ecosystems typically have limited capacity to buffer acidity that may be deposited with airborne contaminants such as nitrogen and sulfur. Although efforts to reduce nitrogen and sulfur emissions are ongoing nationally for large point sources like powerplants (U.S. Environmental Protection Agency, 2006c), continuing growth and development likely will result in greater numbers of large and small emissions sources (such as mobile sources with gasoline and diesel engines) in the region (U.S. Environmental Protection Agency, 2006d). Atmospheric input of mercury to sensitive areas may affect aquatic and terrestrial plant and wildlife populations and has been associated with fish advisories for surface-water bodies in all States in the study area except Wyoming (U.S. Environmental Protection Agency, 2001; 2004).

Although several watershed-scale studies have investigated anthropogenic deposition in small headwater basins in the Rocky Mountain region (Turk and Campbell, 1987; Caine and Thurman, 1990; Baron, 1992; Reuss and others, 1993; Campbell and others, 1995; Williams and others, 1996; and Burns, 2002), regional-scale atmospheric deposition data are sparse. The National Atmospheric Deposition Program (NADP) provides nationwide estimates of atmospheric deposition (Nilles, 2000; National Atmospheric Deposition Program, 2004a). However, coverage for highelevation areas (greater than 2,400 meters [m]) in the Rocky Mountains is limited. Although 13 NADP sites monitor 
atmospheric deposition above $2400 \mathrm{~m}$ in Colorado, few sites are operated in the high-elevation areas of Idaho, Montana, Wyoming, and New Mexico, where snowpacks persist with negligible melt through the snowfall season. These high-elevation snowpacks are important because they may accumulate two to three times or more of the annual precipitation measured at lower elevations where regular monitoring is more feasible.

To gain a better understanding of atmospheric deposition at high elevation in the Rocky Mountains, the U.S. Geological Survey (USGS), in cooperation with the National Park Service, U.S. Department of Agriculture, Forest Service, and other organizations established a network of more than 50 snow-sampling sites in the Rocky Mountain region. Sites in the network have been sampled annually since 1993.

\section{Purpose and Scope}

The purpose of this report is to present the 2004 snowpack chemistry data as an annual update and for comparison to previous years (Ingersoll and others, 2003, 2005; Mast and others, 2001; Turk and others, 2001).

\section{Study Area}

Snow-sampling sites in Montana, Idaho, Wyoming, Colorado, and New Mexico generally are located near the Continental Divide in national forests or national parks. Sampling sites were chosen at locations with limited human activity or emissions from local residential, commercial, or industrial activities to enable detection of regional emissions signals that may affect deposition hundreds of kilometers downwind. Sites were located at least $30 \mathrm{~m}$ away from plowed roadways to minimize contamination from vehicular traffic. Sites in Colorado and New Mexico range in elevation from about 2,700 to 3,400 m; sites in Idaho, Wyoming and Montana typically are lower at about 1,800 to $2,700 \mathrm{~m}$. At these elevations, seasonal snowpacks accumulate throughout the winter, and substantial snowmelt does not occur until spring runoff begins in March, April, or May. The seasonal snowpacks melt entirely each summer at all sites in the network, so resampling snowfall from previous years is avoided. As latitude increases along the Continental Divide, the elevation at which seasonal snowpacks develop generally decreases.

\section{Acknowledgments}

The authors are grateful for the cooperation from numerous individuals from many organizations including the National Park Service and the U.S. Department of Agriculture, Forest Service (USDA). The authors also owe thanks to many individuals for their assistance with logistical support, field work, and analytical processing, including Stan Bones,
Cindy Bosco, Jay Dorr, Dan Fagre, Ben Glass, Bob Hammer, Mary Hektner, Karen Holzer, Nan Ingersoll, Cyndi Kester, Craig McClure, Lisa McKeon, Rick Neam, Gary Nelson, Ted Porwoll, Blaze Reardon, Don Rosenberry, Orville Rosenberry, John Sacklin, Mark Story, and Terry Svalberg. Special thanks also are due to the following ski areas who provided generous logistical support: The Big Mountain, Big Sky, Loveland, Showdown, Snow Bowl, Taos Ski Valley, and Teton Village.

\section{Study Methods}

The U.S. Geological Survey has developed methods for measuring physical and chemical properties of seasonal snowpacks. Detailed descriptions of sample-collection and analytical methods are reported in previous publications (Ingersoll and others, 2002; Mast and others, 2001; Turk and others, 2001).

\section{Data Collection}

Snowpack samples were collected at 65 sites in 2004 including 48 (of 50) long-term sampling sites that have been sampled annually since 1993. Early melt in March prevented sampling at two long-term sites (Deadman Pass and Divide Peak). An additional 15 sites were added since 1993 to expand the coverage of the network in Colorado, Idaho, Montana, and Wyoming. Where feasible, snow-sampling sites were collocated with snowpack-telemetry (SNOTEL) instrument sites where measurements of snow-water equivalent (SWE) were recorded daily by the USDA, Natural Resources Conservation Service (National Resources Conservation Service, 2004). Snowpack samples were collected from late February to early April just before the time of the annual maximum snow depth. Samples were collected from snowpits (fig. 1) in small clearings in forests where uniform snow cover appeared to be free of human disturbance, excessive tree litter, and animal activity. Sampling protocols include observations and measurements of physical snow properties to ensure the snowpack had not been contaminated or flushed with early-season runoff.

\section{Analytical Methods}

Concentrations of major ions and trace constituents were determined from snow melted in 8-liter (L) Teflon bags containing a single, depth-integrated, composite sample from each snowpit. Method detection limits were 1.0 microequivalent per liter ( $\mu \mathrm{eq} / \mathrm{L}$ ) for acid neutralizing capacity (ANC), 0.2 to $2.0 \mu \mathrm{eq} / \mathrm{L}$ for major ions (calcium, 1.2; magnesium, 0.6; sodium, 2.0; potassium, 0.4; ammonium, 0.5; chloride, 0.5; sulfate, 0.3 ; nitrate, 0.2$), 0.15$ milligrams per liter $(\mathrm{mg} / \mathrm{L})$ for dissolved organic carbon, and 0.4 nanograms per liter (ng/L) for mercury. Analytical laboratory methods and qualityassurance procedures for analyses of major-ion and mercury concentrations, and stable sulfur isotope ratios are described in 


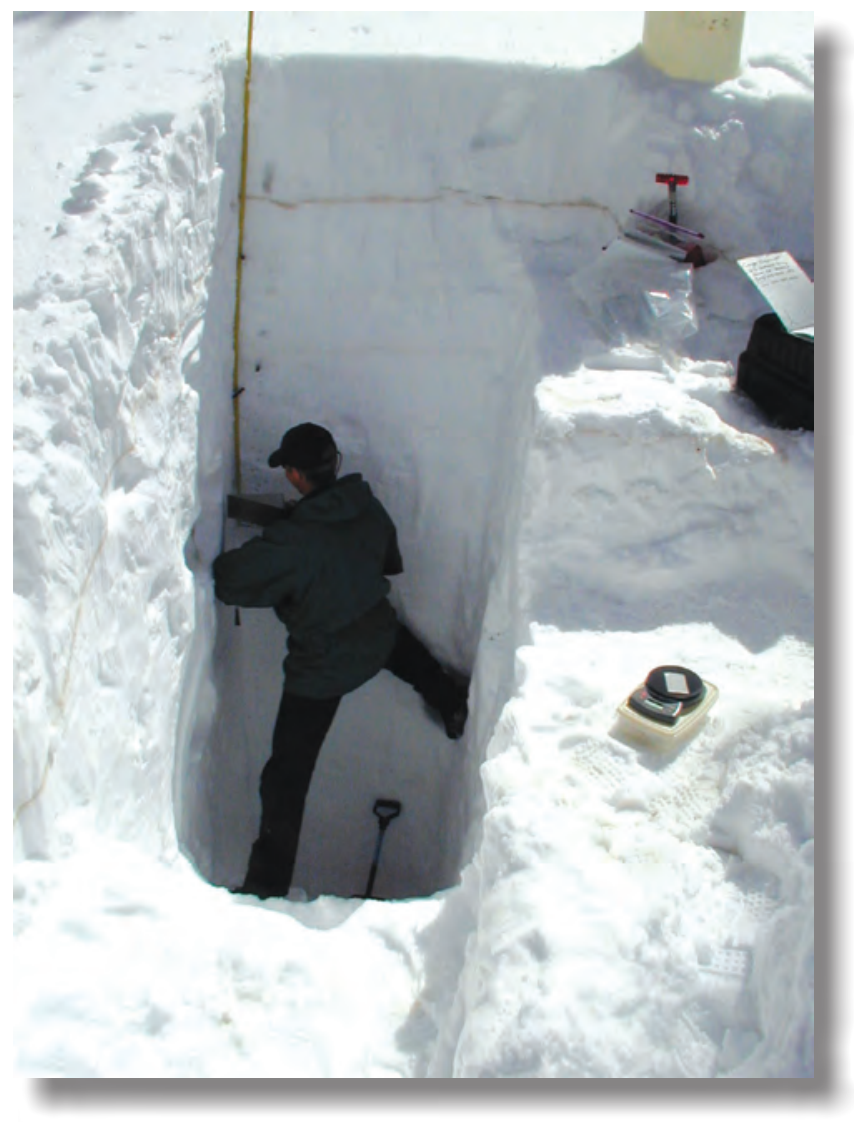

Figure 1. Worker collecting samples in a snowpit. (Photograph by George P. Ingersoll, U.S. Geological Survey.)

Ingersoll and others (2002), Mast and others (2001), and Turk and others (2001). Additional information including interlaboratory comparisons of USGS standard reference samples can be found at http://bqs.usgs.gov/srs\#contacts.

\section{Snowpack Chemistry}

\section{Water Content}

When snow samples were collected during February, March, and April, the snowpack water content, referred to as "snow-water equivalent (SWE)," was near average to well below average throughout most of the Rocky Mountain region. Snow depths at sampling sites ranged from 57.0 to 303.0 centimeters $(\mathrm{cm})$, with a mean depth of $148.8 \mathrm{~cm}$. SWE measured for full snowpacks ranged from about 15.2 to $109.6 \mathrm{~cm}$ with a mean of $47.9 \mathrm{~cm}$. On March 1 2004, SWE in regional snowpacks sampled ranged from 70 to 108 percent of the 30-year average, and by April 1, SWE decreased to 50 to 89 percent. When compared to 30 -year averages for
1971-2000 (as measured at SNOTEL sites representative of basins where snow sampling sites were located), SWE accumulations on April 1 were 76 to 89 percent in Montana, 64 to 81 percent in Idaho, 70 to 80 percent in Wyoming, 60 to 79 percent in Colorado, and 50 to 64 percent in New Mexico (National Resources Conservation Service, 2004).

\section{Chemistry}

Chemical data for 2004, including ANC, laboratory $\mathrm{pH}$, average concentrations of major ions, dissolved organic carbon, total mercury, stable sulfur isotope ratios, ionic charge balances, and quality-assurance blanks and replicates are presented in tables 1 to 3. Snow-sampling sites listed by State in table 1 also are referenced by their site numbers in figures, and site numbers appear in parentheses directly after the site names in the following text. Readers should consult table 1 for clarification of concentration values in areas where several colored circles indicating sample concentrations overlap (such as in Colorado).

Concentrations of ammonium ranged from $<0.5 \mu \mathrm{eq} / \mathrm{L}$ at Banner Summit, Idaho (18) to $12.7 \mu \mathrm{eq} / \mathrm{L}$ at Niwot SNOTEL, in northern Colorado (55) with an average of $4.9 \mu \mathrm{eq} / \mathrm{L}$ (fig. 2, table 1). At sites reported during 1993-2004, snowpack ammonium concentrations for 2004 were lower than average concentrations for the 12-year period at 61 percent of sites in the region. Ammonium concentrations tended to be lowest at sites surrounded by mountain forests with limited agricultural land use, and highest near areas of large-scale agricultural activity at lower elevations. The second highest ammonium concentration occurred at Lionshead, Montana (8) $(9.6 \mu \mathrm{eq} / \mathrm{L})$. The third, fourth, and fifth highest concentrations also were observed in northern Colorado at Loch Vale Forest (47) $(9.3 \mu \mathrm{eq} / \mathrm{L})$, University Camp (62) $(9.0 \mu \mathrm{eq} / \mathrm{L})$, and westcentral Montana at Spring Gulch (16) $(8.7 \mu \mathrm{eq} / \mathrm{L})$, respectively. The high ammonium concentration in southwestern Montana at the Lionshead site is consistent with previous years (2001-2003), but the occurrence of three of the four highest values for 2004 at Niwot SNOTEL and nearby Loch Vale Forest and University Camp in Colorado is unusual. Ammonium concentrations at those three sites were higher than average during the past 11 years. The 11 -year averages for the three sites are: $9.3 \mu \mathrm{eq} / \mathrm{L}$ at Niwot SNOTEL; $5.2 \mu \mathrm{eq} / \mathrm{L}$ at Loch Vale Forest; and $6.9 \mu \mathrm{eq} / \mathrm{L}$ at University Camp. The Lionshead site is located near a mountain pass where prevailing westerly winds funnel air masses over the Continental Divide from west to east (Thompson and others, 1993). Storms originating out of the Southwest may travel over large agricultural areas of Idaho, where large-scale application of fertilizer occurs, before reaching the site. The site at West Yellowstone, Montana (17), located about 16 kilometers (km) east of the Lionshead site in Yellowstone National Park, had the 8th highest ammonium concentration $(7.5 \mu \mathrm{eq} / \mathrm{L})$. Similarly, in Colorado, elevated ammonium concentrations at Niwot SNOTEL and the two nearby sites, including one in Rocky Mountain 
Table 1. Snow depth and snow-water equivalent; ANC and laboratory pH; average concentrations of major ions, dissolved organic carbon, mercury, and sulfur-34 ; and ionic charge balances from analyses of the 2004 snowpack.

[Snow depth and snow-water equivalent (SWE) are in centimeters. Acid neutralizing capacity (ANC), hydrogen $\left(\mathrm{H}^{+}\right)$, calcium $\left(\mathrm{Ca}^{2+}\right)$, magnesium $\left(\mathrm{Mg}^{2+}\right)$, sodium $\left(\mathrm{Na}^{+}\right)$, potassium $\left(\mathrm{K}^{+}\right)$, ammonium $\left(\mathrm{NH}_{4}^{+}\right)$, chloride $\left(\mathrm{Cl}^{-}\right)$, sulfate $\left(\mathrm{SO}_{4}^{2-}\right)$, nitrate $\left(\mathrm{NO}_{3}^{-}\right)$are in microequivalents per liter; dissolved organic carbon (DOC) values expressed in milligrams per liter. Mercury (Hg) expressed as nanograms per liter from whole-water samples. Sulfur-34 $\left(\delta^{34} \mathrm{~S}\right)$, per mil; “-”, not analyzed; "<", below detection limits. Ionic balances are percentages. na, not applicable]

\begin{tabular}{|c|c|c|c|c|c|c|c|c|c|c|c|c|c|c|c|c|c|c|}
\hline $\begin{array}{c}\text { Site } \\
\text { number } \\
\text { shown } \\
\text { in } \\
\text { figures } \\
2-6\end{array}$ & $\begin{array}{c}\text { Site } \\
\text { name }\end{array}$ & $\begin{array}{l}\text { Snow } \\
\text { depth }\end{array}$ & SWE & ANC & pH & $\mathrm{H+}$ & $\mathrm{Ca}^{2+}$ & $\mathbf{M g}^{+}$ & $\mathrm{Na}^{+}$ & $\mathbf{K}^{+}$ & $\mathrm{NH}_{4}^{+}$ & $\mathrm{Cl}^{-}$ & $\mathrm{SO}_{4}{ }^{2-}$ & $\mathrm{NO}_{3}^{-}$ & DOC & $\mathrm{Hg}$ & $\delta^{34} \mathbf{S}$ & $\begin{array}{l}1,2 \text { lonic } \\
\text { balance }\end{array}$ \\
\hline 1 & Apgar Lookout, Mont. & 177 & 61.0 & -10.2 & 5.09 & 8.1 & 2.6 & $<0.6$ & $<2.0$ & 0.4 & 5.0 & 2.0 & 4.3 & 7.7 & 0.5 & 1.7 & - & 7.24 \\
\hline 2 & Big Mountain, Mont. & 252 & 73.8 & -13.5 & 5.12 & 7.6 & $<1.2$ & $<0.6$ & $<2.0$ & 1.2 & 3.3 & 1.5 & 3.6 & 5.3 & 0.6 & 1.7 & 5.6 & 7.99 \\
\hline 3 & Big Sky, Mont. & 77 & 22.6 & -4.6 & 5.43 & 3.7 & 2.7 & $<0.6$ & $<2.0$ & $<0.4$ & 3.0 & 0.6 & 3.0 & 4.5 & 0.2 & 0.9 & - & 8.03 \\
\hline 4 & Chief Joseph Pass, Mont. & 176 & 48.1 & -8.0 & 5.20 & 6.3 & 2.3 & 0.8 & $<2.0$ & 1.4 & 2.4 & 1.3 & 2.5 & 3.0 & 1.2 & 4.2 & 2.9 & 31.89 \\
\hline 5 & Daisy Pass , Mont. & 220 & 72.6 & -5.4 & 5.38 & 4.2 & 3.5 & 1.0 & $<2.0$ & 1.2 & 5.1 & 0.7 & 3.8 & 4.9 & 0.9 & 1.1 & - & 22.74 \\
\hline 6 & Granite Pass, Mont. & 222 & 64.3 & -15.1 & 4.92 & 12.0 & 1.9 & 0.6 & $<2.0$ & 1.1 & 2.1 & 1.3 & 2.0 & 2.8 & 0.7 & 6.3 & - & 48.51 \\
\hline 7 & Kings Hill, Mont. & 128 & 42.0 & -10.2 & 5.11 & 7.8 & 4.2 & 0.7 & $<2.0$ & 1.1 & 4.8 & 0.8 & 4.1 & 7.1 & 0.8 & 3.0 & 8.0 & 21.55 \\
\hline 8 & Lionshead, Mont. & 170 & - & -10.9 & 5.04 & 9.1 & 4.1 & 1.0 & $<2.0$ & 2.0 & 9.6 & 1.6 & 7.6 & 10.0 & 0.9 & 2.1 & - & 14.62 \\
\hline 9 & Monida Pass, Mont. & 57 & 15.2 & -4.8 & 5.32 & 4.8 & 4.2 & $<0.6$ & 2.4 & $<0.4$ & 4.4 & 1.5 & 4.3 & 7.5 & 0.3 & 0.9 & - & 8.74 \\
\hline 10 & Mount Belmont, Mont. & 68 & 21.9 & -5.5 & 5.27 & 5.4 & 7.3 & 1.9 & 3.0 & 3.2 & 5.9 & 2.5 & 5.1 & 8.3 & 1.8 & 3.9 & - & 25.33 \\
\hline 11 & Noisy Basin, Mont. & 303 & 82.1 & -7.0 & 5.10 & 7.9 & $<1.2$ & $<0.6$ & $<2.0$ & $<0.4$ & 3.1 & 0.6 & 3.2 & 5.5 & 0.3 & 1.5 & - & 8.33 \\
\hline 13 & Red Mountain, Mont. & 136 & 34.9 & -10.8 & 5.05 & 8.9 & 5.6 & 0.8 & 2.6 & 0.4 & 6.0 & 1.6 & 5.5 & 6.4 & 0.6 & 1.5 & - & 28.76 \\
\hline 14 & Snow Bowl, Mont. & 198 & 56.8 & -24.4 & 4.73 & 18.6 & 1.6 & $<0.6$ & $<2.0$ & 0.9 & 1.6 & 1.0 & 2.4 & 3.4 & 0.8 & 3.6 & - & 54.09 \\
\hline 15 & Snyder Lake, Mont. & 137 & 43.3 & -5.4 & 5.26 & 5.495 & $<1.2$ & $<0.6$ & $<2.0$ & 0.5 & 2.9 & 1.5 & 2.7 & 5.3 & 0.5 & 3.2 & - & -3.17 \\
\hline 16 & Spring Gulch, Mont. & 68 & 21.6 & -14.3 & 4.95 & 11.2 & 4.4 & 1.1 & $<2.0$ & 3.3 & 8.7 & 0.7 & 6.5 & 9.5 & 1.3 & 2.0 & - & 26.24 \\
\hline 17 & West Yellowstone, Mont. & 90 & 23.5 & -2.8 & 5.20 & 6.3 & 5.0 & 1.2 & $<2.0$ & 0.8 & 7.5 & 1.4 & 4.8 & 11.7 & 1.0 & 1.4 & - & 7.54 \\
\hline 18 & Banner Summit, Idaho & 173 & 63.8 & 12.5 & 6.12 & 0.8 & 15.4 & 2.3 & 32.7 & 0.5 & $<0.5$ & 12.5 & 15.7 & 3.0 & 0.3 & 4.1 & 7.7 & 8.43 \\
\hline 19 & Galena Summit, Idaho & 145 & 43.8 & 1.9 & 5.52 & 3.0 & 5.0 & 1.0 & 10.7 & 0.6 & 2.8 & 4.0 & 6.8 & 3.0 & - & 2.7 & 7.6 & 18.83 \\
\hline 20 & Brooklyn Lake, Wyo. & 147 & 54.1 & -1.4 & 5.23 & 5.9 & 13.1 & 3.5 & $<2.0$ & 5.1 & 6.4 & 1.7 & 9.8 & 12.4 & 1.4 & 14.0 & 6.3 & 17.39 \\
\hline 21 & Canyon, Wyo. & 111 & 29.1 & -16.6 & 5.12 & 7.6 & 1.7 & $<0.6$ & $<2.0$ & $<0.4$ & 3.3 & 0.7 & 2.6 & 6.1 & $<0.15$ & 0.5 & - & 14.25 \\
\hline 22 & Elkhart Park, Wyo. & 110 & 35.6 & -13.1 & 5.06 & 8.7 & 3.4 & 0.7 & $<2.0$ & $<0.4$ & 3.3 & 0.9 & 5.5 & 7.4 & 0.6 & 0.4 & 5.9 & 7.77 \\
\hline 23 & Four Mile Meadow, Wyo. & 81 & 23.1 & -4.8 & 5.28 & 5.2 & 3.4 & 0.8 & $<2.0$ & $<0.4$ & 3.4 & 0.8 & 2.3 & 6.3 & 0.5 & 0.8 & - & 15.57 \\
\hline 24 & Garnet Canyon, Wyo. & 119 & 47.9 & 2.2 & 5.64 & 2.3 & 9.7 & 2.5 & $<2.0$ & 2.8 & 4.9 & 1.0 & 5.3 & 7.5 & 1.4 & 2.7 & - & 16.15 \\
\hline 25 & Gypsum Creek, Wyo. & 91 & 23.7 & -7.1 & 5.35 & 4.5 & 4.6 & 1.5 & $<2.0$ & 0.4 & 2.5 & 1.3 & 3.1 & 6.9 & 0.6 & 0.9 & - & 9.05 \\
\hline 26 & Lake, Wyo. & 105 & 28.2 & -5.5 & 5.42 & 3.8 & 2.5 & $<0.6$ & $<2.0$ & $<0.4$ & 5.1 & 0.6 & 2.8 & 5.7 & 0.5 & 2.1 & - & 11.25 \\
\hline
\end{tabular}


Table 1. Snow depth and snow-water equivalent; ANC and laboratory pH; average concentrations of major ions, dissolved organic carbon, mercury, and sulfur-34; and ionic charge balances from analyses of the 2004 snowpack.-Continued

[Snow depth and snow-water equivalent ( $\mathrm{SWE})$ are in centimeters. Acid neutralizing capacity $(\mathrm{ANC})$, hydrogen $\left(\mathrm{H}^{+}\right)$, calcium $\left(\mathrm{Ca}^{2+}\right)$, magnesium $\left(\mathrm{Mg}^{2+}\right)$, sodium $\left(\mathrm{Na}^{+}\right)$, potassium $\left(\mathrm{K}^{+}\right)$, ammonium $\left(\mathrm{NH}_{4}^{+}\right)$, chloride $\left(\mathrm{Cl}^{-}\right)$, sulfate $\left(\mathrm{SO}_{4}^{2-}\right)$, nitrate $\left(\mathrm{NO}^{-}\right)$are in microequivalents per liter; dissolved organic carbon (DOC) values expressed in milligrams per liter. Mercury $\left(\mathrm{Hg}^{-}\right)$ expressed as nanograms per liter from whole-water samples. Sulfur-34 $\left(\delta^{34} \mathrm{~S}\right)$, per mil; “-”, not analyzed; "<”, below detection limits. Ionic balances are percentages. na, not applicable]

\begin{tabular}{|c|c|c|c|c|c|c|c|c|c|c|c|c|c|c|c|c|c|c|}
\hline $\begin{array}{c}\text { Site } \\
\text { number } \\
\text { shown } \\
\text { in } \\
\text { figures } \\
2-6\end{array}$ & $\begin{array}{l}\text { Site } \\
\text { name }\end{array}$ & $\begin{array}{l}\text { Snow } \\
\text { depth }\end{array}$ & SWE & ANC & $\mathrm{pH}$ & $\mathbf{H +}$ & $\mathrm{Ca}^{2+}$ & $\mathbf{M g}^{+}$ & $\mathrm{Na}^{+}$ & $\mathbf{K}^{+}$ & $\mathrm{NH}_{4}^{+}$ & $\mathrm{Cl}^{-}$ & $\mathrm{SO}_{4}{ }^{2-}$ & $\mathrm{NO}_{3}^{-}$ & DOC & $\mathrm{Hg}$ & $\delta^{34} \mathbf{S}$ & $\begin{array}{l}\text { 1,2lonic } \\
\text { balance }\end{array}$ \\
\hline 29 & $\begin{array}{l}\text { Old Faithful Fire Road, } \\
\text { Wyo. }\end{array}$ & 123 & 37.1 & 1.4 & 5.63 & 2.3 & 4.3 & 0.6 & $<2.0$ & $<0.4$ & 5.6 & 1.6 & 3.5 & 6.3 & 0.5 & 1.1 & - & -0.02 \\
\hline 30 & $\begin{array}{l}\text { Rendevous Mountain, } \\
\text { Wyo. }\end{array}$ & 243 & 90.0 & -5.5 & 5.48 & 3.3 & 3.1 & 0.7 & $<2.0$ & 0.5 & 4.5 & 0.7 & 3.8 & 4.5 & $<0.15$ & 0.7 & - & 14.09 \\
\hline 31 & South Pass, Wyo. & 122 & 41.1 & -10.0 & 5.16 & 6.9 & 7.0 & 1.3 & $<2.0$ & 1.8 & 4.6 & 0.8 & 8.0 & 8.4 & 0.9 & 1.8 & - & 11.51 \\
\hline 32 & Sylvan Lake, Wyo. & 142 & 46.3 & -6.6 & 5.42 & 3.8 & 2.8 & $<0.6$ & $<2.0$ & 0.5 & 4.0 & $<0.5$ & 3.1 & 5.0 & 0.5 & 1.3 & - & 15.44 \\
\hline 33 & Teton Pass, Wyo. & 199 & 48.1 & 6.7 & 6.03 & 0.9 & 8.6 & 2.9 & $<2.0$ & 2.0 & 5.6 & 1.4 & 4.4 & 6.5 & 0.7 & 2.5 & - & 2.57 \\
\hline 34 & Togwotee Pass, Wyo. & 138 & 40.2 & -0.5 & 5.69 & 2.0 & 4.6 & 0.8 & $<2.0$ & 0.8 & 3.4 & 0.7 & 3.6 & 5.0 & 0.4 & 1.5 & - & 11.21 \\
\hline 35 & Twenty-one Mile, Wyo. & 134 & 36.2 & -12.2 & 5.06 & 8.7 & 6.0 & 1.0 & $<2.0$ & 2.8 & 5.5 & 1.4 & 4.3 & 7.4 & 1.3 & 1.4 & - & 29.63 \\
\hline 36 & Berthoud Pass, Colo. & 166 & 50.0 & -13.2 & 5.27 & 5.4 & 6.3 & 1.3 & $<2.0$ & 0.9 & 5.7 & 1.5 & 4.5 & 9.7 & 0.6 & 4.0 & - & 11.09 \\
\hline 38 & Buffalo Pass, Colo. & 279 & 102.5 & -14.0 & 5.05 & 9.1 & 6.2 & 1.2 & $<2.0$ & 1.2 & 6.6 & 1.2 & 9.6 & 12.3 & 0.6 & 2.1 & - & 2.76 \\
\hline 39 & Cameron Pass, Colo. & 132 & 34.5 & -11.3 & 4.97 & 10.7 & 5.8 & 1.0 & 2.1 & 2.0 & 4.9 & 2.8 & 6.7 & 11.8 & 1.0 & 2.6 & - & 10.95 \\
\hline 40 & Dry Lake, Colo. & 127 & 46.7 & -12.0 & 4.97 & 10.7 & 6.7 & 1.1 & $<2.0$ & $<0.4$ & 3.4 & 0.9 & 6.9 & 17.0 & 0.4 & 1.1 & - & -6.17 \\
\hline 41 & Dunckley Pass, Colo. & 174 & 59.5 & -3.1 & 5.69 & 2.0 & 14.4 & 2.5 & $<2.0$ & 2.2 & 5.0 & 1.0 & 5.7 & 11.5 & 0.6 & 1.2 & - & 18.10 \\
\hline 42 & Elk River, Colo. & 118 & 43.2 & -8.9 & 5.18 & 6.6 & 11.0 & 2.0 & $<2.0$ & 1.0 & 4.4 & 0.7 & 7.5 & 14.4 & 0.4 & 1.7 & - & 4.73 \\
\hline 43 & Fremont Pass, Colo. & 120 & 28.8 & -2.7 & 5.32 & 4.8 & 6.1 & 1.3 & $<2.0$ & 2.9 & 4.8 & 0.9 & 4.2 & 9.0 & 0.8 & 2.5 & 4.4 & 16.98 \\
\hline 44 & Grand Mesa, Colo. & 101 & 37.0 & 2.5 & 5.58 & 2.6 & 27.0 & 5.0 & $<2.0$ & 7.8 & 6.4 & 2.6 & 10.9 & 13.9 & 2.2 & 5.3 & - & 24.05 \\
\hline 45 & Lake Irene Forest, Colo. & 145 & 44.3 & -9.7 & 5.16 & 7.0 & 9.1 & 1.7 & $<2.0$ & 2.3 & 4.8 & 0.9 & 7.2 & 12.6 & 1.0 & 2.6 & - & 9.44 \\
\hline 46 & $\begin{array}{l}\text { Lake Irene Meadow, } \\
\text { Colo. }\end{array}$ & 105 & 35.1 & -7.8 & 5.23 & 5.9 & 2.6 & $<0.6$ & $<2.0$ & 0.4 & 3.5 & 0.5 & 2.6 & 8.2 & 0.3 & 1.4 & - & 4.09 \\
\hline 47 & Loch Vale Forest, Colo. & 219 & 73.3 & -11.9 & 5.20 & 6.3 & 9.8 & 1.5 & $<2.0$ & 2.1 & 9.3 & 0.9 & 8.0 & 14.4 & 0.7 & 3.9 & 4.5 & 11.05 \\
\hline 48 & Loch Vale Meadow, Colo. & 302 & 109.6 & -5.4 & 5.29 & 5.1 & 7.1 & 1.2 & $<2.0$ & 0.9 & 5.7 & 0.8 & 5.8 & 11.5 & 0.4 & 2.0 & - & 4.92 \\
\hline 49 & Loveland Pass, Colo. & 144 & 41.9 & -13.1 & 5.17 & 6.8 & 3.7 & 1.2 & $<2.0$ & $<0.4$ & 5.2 & 2.3 & 3.8 & 8.4 & 0.4 & 0.8 & - & 7.94 \\
\hline
\end{tabular}


Table 1. Snow depth and snow-water equivalent; ANC and laboratory pH; average concentrations of major ions, dissolved organic carbon, mercury, and sulfur-34 ; and ionic charge balances from analyses of the 2004 snowpack. - Continued

[Snow depth and snow-water equivalent (SWE) are in centimeters. Acid neutralizing capacity (ANC), hydrogen $\left(\mathrm{H}^{+}\right)$, calcium $\left(\mathrm{Ca}^{2+}\right)$, magnesium $\left(\mathrm{Mg}^{2+}\right)$, sodium $\left(\mathrm{Na}^{+}\right)$, potassium $\left(\mathrm{K}^{+}\right)$, ammonium $\left(\mathrm{NH}_{4}^{+}\right)$, chloride $(\mathrm{Cl}-)$, sulfate $\left(\mathrm{SO}_{4}^{2-}\right)$, nitrate $\left(\mathrm{NO}_{3}^{-}\right)$are in microequivalents per liter; dissolved organic carbon (DOC) values expressed in milligrams per liter. Mercury (Hg) expressed as nanograms per liter from whole-water samples. Sulfur-34 $\left(\delta^{34} \mathrm{~S}\right)$, per mil; “-”, not analyzed; "<", below detection limits. Ionic balances are percentages. na, not applicable]

\begin{tabular}{|c|c|c|c|c|c|c|c|c|c|c|c|c|c|c|c|c|c|c|}
\hline $\begin{array}{c}\text { Site } \\
\text { number } \\
\text { shown } \\
\text { in } \\
\text { figures } \\
2-6 \\
\end{array}$ & $\begin{array}{c}\text { Site } \\
\text { name }\end{array}$ & $\begin{array}{l}\text { Snow } \\
\text { depth }\end{array}$ & SWE & ANC & pH & $\mathrm{H}+$ & $\mathrm{Ca}^{2+}$ & $\mathbf{M g}^{+}$ & $\mathrm{Na}^{+}$ & $\mathbf{K}^{+}$ & $\mathrm{NH}_{4}^{+}$ & $\mathrm{Cl}^{-}$ & $\mathrm{SO}_{4}^{2-}$ & $\mathrm{NO}_{3}^{-}$ & DOC & $\mathrm{Hg}$ & $\delta^{34} \mathbf{S}$ & $\begin{array}{l}\text { 1,2lonic } \\
\text { balance }\end{array}$ \\
\hline 51 & Mills Lake, Colo. & 170 & 58.0 & -7.8 & 5.25 & 5.623 & 11.6 & 1.71 & $<2.0$ & 0.53 & 8.6 & 0.8 & 8.7 & 16.3 & 0.8 & 2.5 & - & 4.14 \\
\hline 52 & Molas Lake, Colo. & 99 & 37.2 & -2.7 & 5.51 & 3.1 & 9.3 & 1.7 & 3.8 & 1.6 & 3.6 & 4.5 & 4.9 & 9.1 & 1.1 & 1.1 & 3.5 & 11.11 \\
\hline 53 & Monarch Pass, Colo. & 82 & 31.5 & -14.0 & 4.83 & 14.8 & 11.2 & 3.1 & $<2.0$ & 6.1 & 4.0 & 2.1 & 4.7 & 7.8 & 2.0 & 5.7 & - & 45.42 \\
\hline 54 & Ned Wilson, Colo. & 224 & 75.6 & -7.1 & 5.4 & 3.981 & 7.9 & 1.24 & $<2.0$ & $<0.4$ & 3.7 & 0.6 & 3.7 & 7.2 & 0.2 & 2 & - & 19.06 \\
\hline 55 & Niwot SNOTEL, Colo. & 88 & 23.1 & -9.0 & 5.15 & 7.1 & 7.2 & 1.1 & $<2.0$ & 1.8 & 12.7 & 1.1 & 9.1 & 18.0 & 0.6 & 1.1 & - & 2.85 \\
\hline 56 & Phantom Valley, Colo. & 71 & 17.3 & -13.7 & 4.96 & 11.0 & 7.7 & 1.4 & $<2.0$ & 1.5 & 5.5 & 0.8 & 8.7 & 16.3 & 0.8 & 2.7 & - & 2.53 \\
\hline 57 & Rabbit Ears, Colo. & 214 & 76.5 & -16.9 & 4.90 & 12.6 & 4.2 & 0.8 & $<2.0$ & 0.7 & 4.9 & 0.6 & 7.9 & 13.0 & 0.2 & 0.9 & - & 4.00 \\
\hline 58 & Red Mountain Pass, Colo. & 140 & 49.4 & 1.3 & 5.79 & 1.6 & 9.2 & 1.0 & 4.1 & 0.4 & 3.2 & 4.3 & 3.5 & 6.5 & 0.3 & 1.3 & - & 11.42 \\
\hline 59 & $\begin{array}{l}\text { Ripple Creek NADP, } \\
\text { Colo. }\end{array}$ & 220 & 82.9 & -8.3 & 5.42 & 3.801 & 8.6 & 1.5 & $<2.0$ & 0.58 & 4.3 & 0.8 & 4.7 & 10.9 & 0.3 & 1.6 & - & 6.84 \\
\hline 60 & Slumgullion Pass, Colo. & 81 & 18.6 & -11.5 & 5.02 & 9.5 & 7.9 & 2.8 & $<2.0$ & 6.4 & 3.3 & 1.4 & 4.8 & 7.4 & 2.2 & 3.8 & 5.0 & 37.88 \\
\hline 61 & Sunlight Peak, Colo. & 133 & 39.2 & -6.7 & 5.26 & 5.5 & 18.5 & 3.9 & $<2.0$ & 5.5 & 6.4 & 1.6 & 8.9 & 14.5 & 2.3 & 4.7 & - & 22.79 \\
\hline 62 & University Camp, Colo. & 184 & 60.5 & -8.8 & 5.21 & 6.2 & 5.9 & 0.9 & $<2.0$ & 1.0 & 9.0 & 0.8 & 7.9 & 12.7 & 0.5 & 0.8 & - & 3.82 \\
\hline 63 & Wolf Creek Pass, Colo. & 187 & 68.0 & -3.6 & 5.27 & 5.4 & 7.1 & 2.0 & $<2.0$ & 5.2 & 7.9 & 2.0 & 9.2 & 11.4 & 1.4 & 4.3 & - & 10.11 \\
\hline 64 & Hopewell, N. Mex. & 87 & 34.1 & -6.1 & 5.23 & 5.9 & 10.9 & 1.8 & $<2.0$ & 2.1 & 4.5 & 1.3 & 6.9 & 11.3 & 1.0 & 4.1 & - & 12.86 \\
\hline \multirow[t]{6}{*}{65} & Taos Ski Valley, N. Mex. & 115 & 36.2 & -12.2 & 5.05 & 8.9 & 4.2 & $<0.6$ & $<2.0$ & 0.9 & 4.3 & 0.7 & 3.8 & 6.6 & 0.7 & 7.5 & 4.1 & 24.96 \\
\hline & Summary Statistics & & & & & & & & & & & & & & & & & \\
\hline & minimum & 57.0 & 15.2 & -24.4 & 4.73 & 0.8 & $<1.2$ & $<0.6$ & $<2.0$ & $<0.4$ & $<0.5$ & $<0.5$ & 2.0 & 2.8 & $<0.15$ & 0.4 & 2.9 & -6.2 \\
\hline & maximum & 303.0 & 109.6 & 12.5 & 6.12 & 18.6 & 27.0 & 5.0 & 32.7 & 7.8 & 12.7 & 12.5 & 15.7 & 18.0 & 2.3 & 14.0 & 8.0 & 54.1 \\
\hline & average $^{3,4}$ & 148.8 & 47.9 & -7.3 & 5.23 & 6.4 & 6.9 & 1.6 & 7.2 & 2.0 & 4.9 & 1.5 & 5.5 & 8.9 & 0.8 & 2.6 & 5.5 & 14.4 \\
\hline & standard deviation & 59.5 & 21.6 & 6.1 & na & 3.3 & 4.5 & 0.9 & 9.9 & 1.8 & 2.1 & 1.6 & 2.6 & 3.8 & 0.5 & 2.2 & 1.7 & 11.7 \\
\hline
\end{tabular}

${ }^{1}$ Percentages for ionic balances are mean values of individual annual calculations of charge balanceof [(total cations - total anions)/(total cations + total anions)] $\mathrm{x} 100$.

${ }^{2}$ Positive ANC are included in total anions; negative ANC are excluded.

${ }^{3}$ The median value for $\mathrm{pH}$ is shown.

${ }^{4}$ Censored values were not included in calculations of averages. 
Table 2 Quality assurance field and laboratory blank results for ammonium, sulfate, nitrate (microequivalents per liter) and mercury (nanograms per liter).

$["<"$, below detection limits. Snow depth and snow-water equivalent; alkalinity and laboratory pH; average concentrations of major ions, dissolved organic carbon, mercury, and sulfur-34; and ionic charge balances from analyses ]

\begin{tabular}{llcccc}
\hline $\begin{array}{c}\text { Site name } \\
\text { and number }\end{array}$ & $\begin{array}{c}\text { Sample } \\
\text { type }\end{array}$ & Ammonium & Sulfate & Nitrate & Mercury \\
\hline Lake Irene Forest (45) & field blank & $<0.5$ & 0.5 & $<0.2$ & 0.6 \\
West Yellowstone (17) & field blank & $<0.5$ & $<0.3$ & $<0.2$ & $<0.4$ \\
USGS laboratory & lab blank & $<0.5$ & $<0.3$ & $<0.2$ & $<0.4$ \\
USGS laboratory & lab blank & $<0.5$ & $<0.3$ & $<0.2$ & $<0.4$ \\
\hline
\end{tabular}

National Park (Loch Vale Forest) and the University Camp site (about $3 \mathrm{~km}$ southwest of Niwot SNOTEL), likely are related to emissions from highly productive agricultural areas of northeastern Colorado. In contrast, ammonium concentrations at two sites in Glacier National Park, in the northern part of the region in northwestern Montana where less agricultural operations exist, were among the 10 lowest concentrations (Oldman Lake (12) $(1.6 \mu \mathrm{eq} / \mathrm{L})$; Snyder Lake (15) $(2.9 \mu \mathrm{eq} / \mathrm{L})$.

Concentrations of nitrate ranged from $2.8 \mu \mathrm{eq} / \mathrm{L}$ at Granite Pass, Montana (6), to $18.0 \mu \mathrm{eq} / \mathrm{L}$ at Niwot SNOTEL, Colorado (55) with an average of $8.9 \mu \mathrm{eq} / \mathrm{L}$ (fig. 3, table 1). Nitrate concentrations in 2004 were lower than the 12-year average for the years 1993-2004 at 53 percent of regional sites, yet Colorado led the region with 9 of the 10 highest concentrations of nitrate detected at sites in the State. Eight of those same 10 sites are within $100 \mathrm{~km}$ of either coal-burning powerplants (located in northwestern Colorado and southern Wyoming) or within 100 to $200 \mathrm{~km}$ of the heavily populated and industrialized Denver area. The four Colorado sites with the highest nitrate concentrations in the region, Niwot SNOTEL (55), Dry Lake (40), Phantom Valley (56) and Mills Lake (51) (table 1) are located between those powerplants and Denver and likely are influenced by snowstorms passing over either of those large emissions sources. Two of those sites are within Rocky Mountain National Park (Phantom Valley and Mills Lake). These elevated concentrations also may reflect greater numbers of mobile and small-emissions sources in the region. Consistently during the 12-year study, lower nitrate concentrations occurred at sites in Wyoming, Idaho, and Montana where there are fewer powerplants and large industrial areas. Nitrate concentrations were among the 10 lowest in the region at the 2 Idaho sites, Banner Summit (18) (3.0 $\mu \mathrm{eq} / \mathrm{L})$ and Galena Summit (19) $(3.0 \mu \mathrm{eq} / \mathrm{L})$, at 1 site in Glacier National Park (Oldman Lake (12) $(4.9 \mu \mathrm{eq} / \mathrm{L}))$, and at 1 site in Yellowstone National Park (Sylvan Lake, Wyoming (32) $(5.0 \mu \mathrm{eq} / \mathrm{L})$ ).

Concentrations of sulfate ranged from $2.0 \mu \mathrm{eq} / \mathrm{L}$ at Granite Pass, Montana (6), to $15.7 \mu \mathrm{eq} / \mathrm{L}$ at Banner Summit, Idaho (18) with an average of $5.5 \mu \mathrm{eq} / \mathrm{L}$ (fig. 4, table 1). Throughout the region, sulfate concentrations in 2004 were lower than the 12 -year average at 84 percent of snowpack sites. Sulfate concentrations generally were lower in the less developed areas of the northern one-half of the region (with the exception of the two sites in central Idaho), and higher in the southern one-half of the region. Sulfate concentrations were among the 10 lowest observed for the region at two sites in Glacier National Park, (Oldman Lake (12) (2.7 $\mu \mathrm{eq} / \mathrm{L})$, and Snyder Lake, Montana (15) (2.7 $\mu \mathrm{eq} / \mathrm{L}))$; at two sites in Yellowstone National Park (Canyon, Wyoming (21) $(2.6 \mu \mathrm{eq} / \mathrm{L})$, and Lake, Wyoming (26) $(2.8 \mu \mathrm{eq} / \mathrm{L}))$; and at one site in Rocky Mountain National Park (Lake Irene Meadow, Colorado (46) $(2.6 \mu \mathrm{eq} / \mathrm{L}))$. This general reduction in sulfate concentrations across most of the Rocky Mountain region is consistent with other monitoring of atmospheric deposition in the Western United States (Lehmann and others, 2005; National Atmospheric Deposition Program, 2006), and with national trends (U.S. Environmental Protection Agency, 2006e).

The highest sulfate concentration in the region was observed in an area where the lowest concentrations in the region were detected throughout the 12-year study. The source of this elevated sulfate is unknown. This high sulfate concentration occurred during a period of reduced emissions from the largest upwind sulfur-emissions sources in Oregon and Washington, according to preliminary data (U.S. Environmental Protection Agency, 2006f). Until 2004, the highest concentrations of sulfate in the region were from sites near large coal-fired power-production facilities in northern Colorado and southern Wyoming. For example, the 12-year average sulfate concentrations for Chief Joseph Pass (4) and Granite Pass (6) in Montana were 2.6 and $2.4 \mu \mathrm{eq} / \mathrm{L}$, respectively. Concentrations of sulfate at Banner Summit (18) also were $2.5 \mu \mathrm{eq} / \mathrm{L}$ in 2002 and $3.3 \mu \mathrm{eq} / \mathrm{L}$ in 2003 , the first 2 years of snow samples were collected at that site. In contrast, average sulfate concentrations for the same period in northern Colorado snowpacks at Buffalo Pass (38) and Dry Lake (40) were 11.4 and $10.8 \mu \mathrm{eq} / \mathrm{L}$, respectively.

All snowpack samples were analyzed for total mercury. Regional mercury concentrations had more complex spatial variability than those observed with ammonium, nitrate, and sulfate. But year-to-year ranges and average concentrations of mercury in snowpacks throughout the region generally are comparable for the 3 years that total mercury has been analyzed in this study. Mercury concentrations decreased 


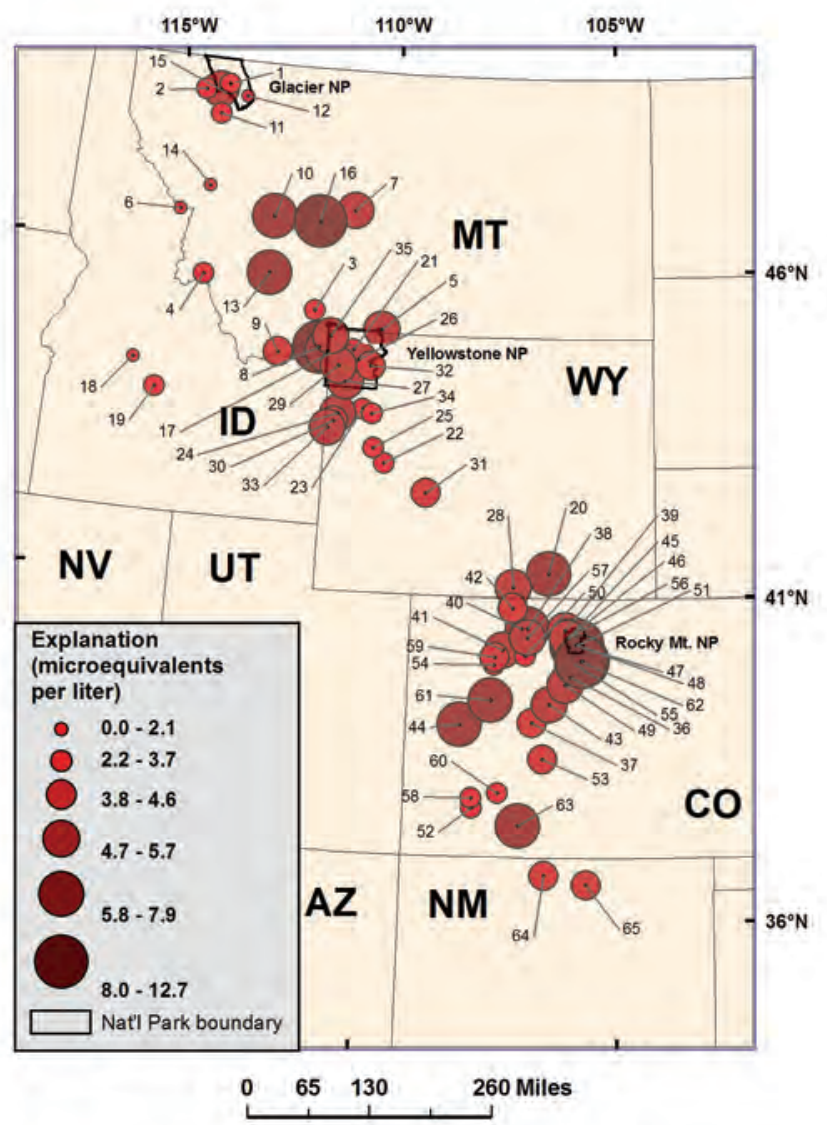

Figure 2. Relative ammonium ion concentrations in snowpacks, 2004.

slightly in 2004 when compared to the 2003 results for the network as a whole, and generally were highest in northern New Mexico, Colorado, and southern Wyoming. Concentrations in 2003 snowpacks ranged from 0.8 nanogram per liter (ng/L) at Lynx Pass, Colorado (50), to $16.3 \mathrm{ng} / \mathrm{L}$ at Brooklyn Lake, Wyoming (20) with an average of $3.0 \mathrm{ng} / \mathrm{L}$ (Ingersoll and others, 2005). Mercury concentrations in 2004 snowpacks ranged from $0.4 \mathrm{ng} / \mathrm{L}$ at Elkhart Park, in northwestern Wyoming (22), to $14.0 \mathrm{ng} / \mathrm{L}$ at Brooklyn Lake in southern Wyoming (20), with an average of $2.6 \mathrm{ng} / \mathrm{L}$ (fig. 5 , table 1). The highest mercury concentration in the region also occurred at Brooklyn Lake in 2003. The second highest concentration of mercury in 2004 was at Taos Ski Valley, in northern New Mexico (65) (7.5 ng/L). Elsewhere in the region, the 2nd lowest concentration was observed in Yellowstone National Park (Canyon, Wyoming (21) $(0.5 \mu \mathrm{eq} / \mathrm{L})$. Although moderate and high concentrations are distributed throughout the region, concentrations at all sites in Yellowstone National Park ranged from 0.5 to $2.1 \mathrm{ng} / \mathrm{L}$ and were below the regional average of $2.6 \mathrm{ng} / \mathrm{L}$. Mercury concentrations in Glacier and Rocky Mountain National Parks mostly were below or near average and ranged from 1.7 to $3.2 \mathrm{ng} / \mathrm{L}$, and 1.4 to $3.9 \mathrm{ng} / \mathrm{L}$, respectively.

These results are in fair agreement with other determinations of total mercury including snowpack- and other precipitation-mercury concentrations collected in 2004 or recent years (Ingersoll and others, 2004, 2005). Mercury concentrations detected in snowpacks in the study area during 2002 and 2003 were within a similar range ( 0.4 to $16.3 \mathrm{ng} / \mathrm{L})$ compared to the 2004 snowpack concentrations. Snowpack-mercury concentrations generally are comparable to weekly precipitation-mercury concentrations at sites in the NADP Mercury Deposition Network (MDN) (National Atmospheric Deposition Program, 2004b). Although MDN sites were sparse in the region, two snowfall-dominated MDN sites at McDonald Lake in Glacier National Park, Montana, and Buffalo Pass, Colorado, were operated during 2004 and offered a comparison to snowpack- mercury concentrations. Weekly total-mercury concentrations measured from October 2003 through April 2004 ranged from 2.4 to $17.4 \mathrm{ng} / \mathrm{L}$ (average = $7.0 \mathrm{ng} / \mathrm{L}$ ) at Buffalo Pass, Colorado. At the mercury monitoring station in Glacier National Park, weekly concentrations ranged from 1.1 to $64.6 \mathrm{ng} / \mathrm{L}$, (average $=8.8 \mathrm{ng} / \mathrm{L})$. If the 


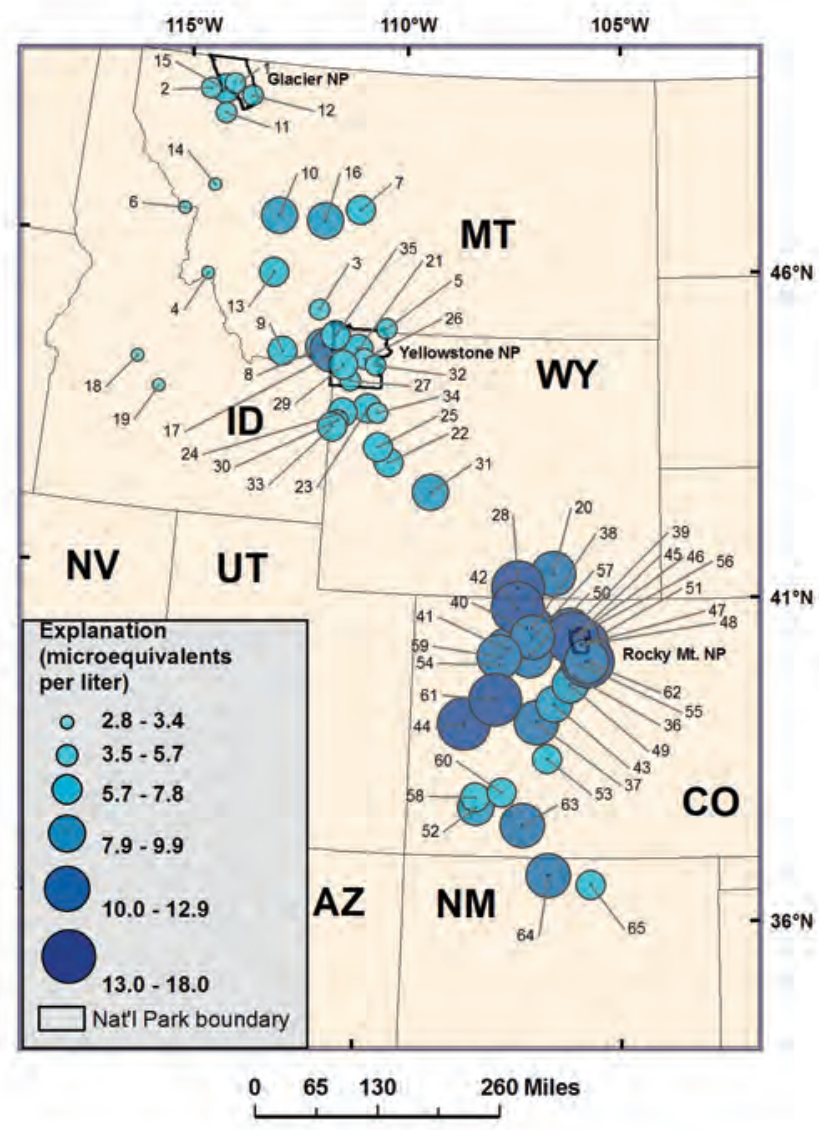

Figure 3. Relative nitrate ion concentrations in snowpacks, 2004.

value of $64.6 \mathrm{ng} / \mathrm{L}$ is discarded as an outlier, the range of values observed was 1.1 to $11.9 \mathrm{ng} / \mathrm{L}$, (average $=4.8 \mathrm{ng} / \mathrm{L}$ ), comparing well with the regional range in this report. For these data, see reference: National Atmospheric Deposition Program, 2004b.

Stable sulfur isotope ratios $\left(\delta^{34} \mathrm{~S}: \delta^{32} \mathrm{~S}\right.$ referred to as $\delta^{34} \mathrm{~S}$ in this report) were determined for a subset of 12 sites in the region (fig. 6, table 1). $\delta^{34} \mathrm{~S}$ ratios ranged from 2.9 per mil at Chief Joseph Pass (4) to 8.0 per mil at Kings Hill, Montana (7), with a average of 5.5 per mil. The regional pattern consisting of the lightest $\delta^{34} \mathrm{~S}$ ratios generally in northern New Mexico and Colorado, and the heaviest values in Wyoming, Idaho, and Montana, as typically seen in recent years, is similar to the results for 2004. One exception is the low value at Chief Joseph Pass that was 2.9 per mil in 2004. The light $\delta^{34} \mathrm{~S}$ ratios of 3.5 to 5.0 at sites in northern New Mexico and Colorado indicate sulfate in atmospheric deposition at the southern part of study area may have been derived from different sources than most of the rest of the study area (except Chief Joseph Pass) where values ranged from 5.6 to 8.0 per mil. Results for $\delta^{34} \mathrm{~S}$ ratios from this study are fairly consistent with previous work (Mast and others, 2001).

\section{Quality Assurance}

Laboratory blanks, field blanks, and field or laboratory replicates composed about 11 percent of sample processing depending upon constituent. Additional laboratory aliquots of barium sulfate precipitate were analyzed for determination of replicate $\delta^{34} \mathrm{~S}$ ratios. Ionic-charge balances were computed as a measure of the quality of the chemical analyses for major ions (table 1).

Two laboratory blanks were analyzed testing the quality of the sample-processing equipment and deionized (DI) water used during analyses. Field blanks were collected at two snow-sampling sites by rinsing sampling tools with DI into Teflon bags after collecting snow samples. Concentrations of all blanks were less than $0.5 \mu \mathrm{eq} / \mathrm{L}$ for major ions (table 2 ). The average total mercury concentration in two blanks and one field blank was below the detection limit of $0.4 \mathrm{ng} / \mathrm{L}$ and $0.6 \mathrm{ng} / \mathrm{L}$ in the fourth blank (field).

Three replicate snow samples were collected at snowsampling sites for evaluation of sampling precision. Replicates were collected from the same snowpit face as the original samples. Percent differences for analytical results for ammo- 


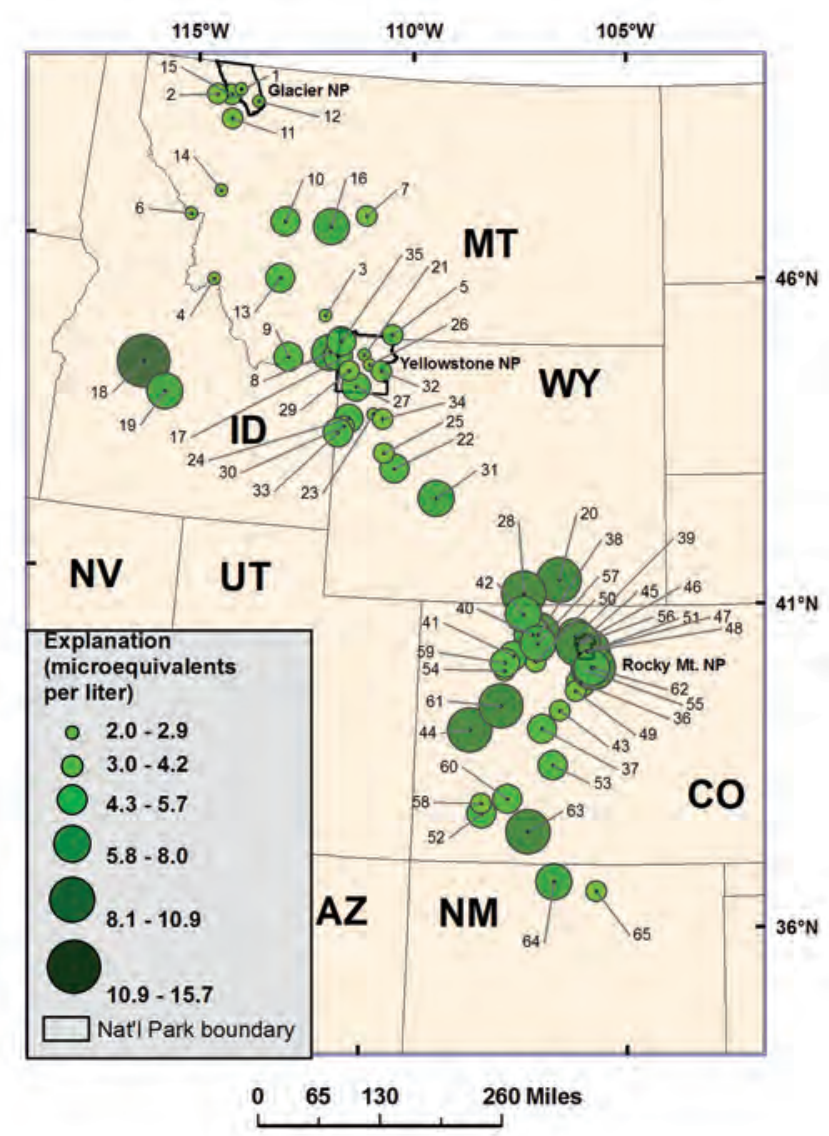

Figure 4. Relative sulfate ion concentrations in snowpacks, 2004.

nium, sulfate, nitrate, and mercury are listed in table 3, and average concentrations of original samples and replicate samples are listed in table 1. Differences between three pairs of original and replicate samples typically were within plus or minus 8 percent for ammonium, sulfate, and nitrate. Differences between mercury samples and replicates of as much as 240.5 percent were due to the inherent variability of total mercury sampling from snowmelt samples. Similar variability was observed in other work (Ingersoll and others, 2005). Percent differences also were more pronounced because of the small concentrations in the calculation (for example, 1.2 compared to $4.1 \mathrm{ng} / \mathrm{L})$.

Ionic charge balance of each major-ion analysis was calculated by dividing the sum of cations (hydrogen ion, calcium, magnesium, sodium, potassium, and ammonium) minus the sum of anions (ANC [>0.0], chloride, nitrate, and sulfate) by the total cations and anions in solution. Ion balances calcu- lated for the 2004 snow chemistry mostly were positive with an average value of 14.4 percent, which indicates an excess of measured cations over anions in solution. This effect was inversely proportional to total cation plus anion concentrations in solution in these very dilute waters and is strongly influenced by small changes in concentrations near detection limits. Such positive ion balances typically have been observed in regional snow chemistry during recent years. Other ionic balances calculated for precipitation chemistry of comparable ionic strength in a separate network yielded somewhat similar deviations (National Atmospheric Deposition Program, 1993). One possible explanation for the positive bias of the ionic balances (table 1) is that organic acids were not included in the calculation. Organic acids such as acetate and formate have been detected in snow in the region in earlier work, with average concentrations of 1.2 and $0.6 \mu \mathrm{eq} / \mathrm{L}$ (Turk and others, 2001). 
Table 3. Quality assurance replicate sample results for ammonium, sulfate, nitrate, mercury, and sulfur-34.

[Snow depth and snow-water equivalent; alkalinity and laboratory pH; average concentrations of major ions, dissolved organic carbon, mercury, and sulfur-34 ; and ionic charge balances from analyses]

\begin{tabular}{|c|c|c|c|c|c|c|c|c|c|c|c|c|c|c|c|c|}
\hline \multirow[b]{2}{*}{$\begin{array}{l}\text { Sampling } \\
\text { site }\end{array}$} & \multicolumn{4}{|c|}{ Ammonium } & \multicolumn{4}{|c|}{ Sulfate } & \multicolumn{4}{|c|}{ Nitrate } & \multicolumn{4}{|c|}{ Mercury } \\
\hline & Sample & $\begin{array}{l}\text { Repli- } \\
\text { cate }\end{array}$ & $\begin{array}{l}\text { Differ- } \\
\text { ence }{ }^{1}\end{array}$ & $\begin{array}{l}\text { Per- } \\
\text { cent }{ }^{2} \\
\text { differ- } \\
\text { ence }\end{array}$ & Sample & $\begin{array}{l}\text { Rep- } \\
\text { licate }\end{array}$ & $\begin{array}{l}\text { Differ- } \\
\text { ence }{ }^{1}\end{array}$ & $\begin{array}{l}\text { Per- } \\
\text { cent }{ }^{2} \\
\text { differ- } \\
\text { ence }\end{array}$ & Sample & $\begin{array}{l}\text { Repli- } \\
\text { cate }\end{array}$ & $\begin{array}{l}\text { Differ- } \\
\text { ence }{ }^{1}\end{array}$ & $\begin{array}{l}\text { Per- } \\
\text { cent }{ }^{2} \\
\text { differ- } \\
\text { ence }\end{array}$ & Sample & $\begin{array}{l}\text { Repli- } \\
\text { cate }\end{array}$ & $\begin{array}{l}\text { Differ- } \\
\text { ence }{ }^{1}\end{array}$ & $\begin{array}{l}\text { Percent }^{2} \\
\text { difference }\end{array}$ \\
\hline Buffalo Pass (38) & 6.5 & 6.7 & 0.2 & 2.6 & 9.4 & 9.8 & 0.4 & 5.1 & 11.9 & 12.6 & 0.7 & 5.7 & 2.1 & 2.0 & -0.1 & -5.3 \\
\hline Lake Irene Forest (45) & 5.0 & 4.7 & -0.3 & -6.2 & 7.2 & 7.2 & 0.0 & 0.0 & 12.4 & 12.7 & 0.4 & 2.8 & 1.2 & 4.1 & 2.9 & 240.5 \\
\hline West Yellowstone in Road $^{3}$ & 8.9 & 8.7 & -0.2 & -2.6 & 5.2 & 4.8 & -0.4 & -7.9 & 8.2 & 8.1 & -0.1 & -1.5 & 1.1 & 1.5 & 0.4 & 43.0 \\
\hline
\end{tabular}

\section{'Replicate - sample.}

${ }^{2}[$ (Replicate - sample)/sample]x 100 .

${ }^{3}$ This sample and replicate pair included for quality-control purposes but are not included in figures 2-6 because local-emissions effects obscure patterns of regional-emissions effects. 


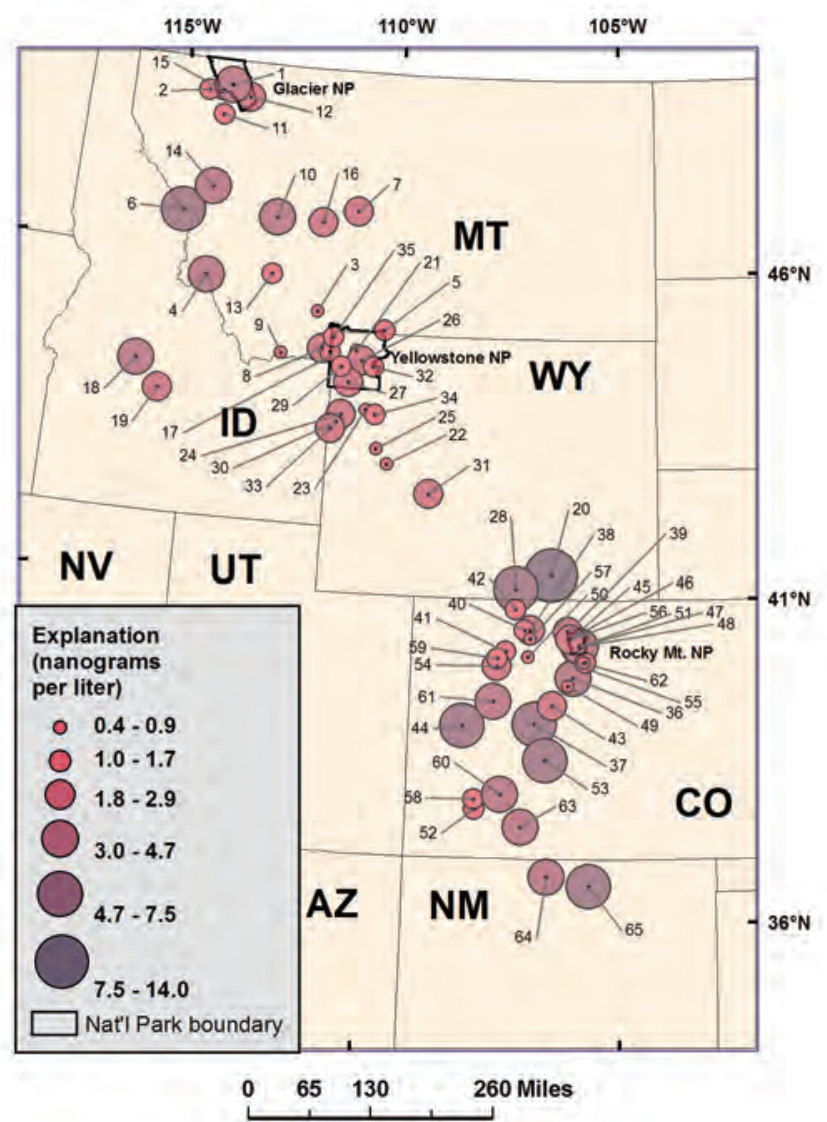

Figure 5. Relative total mercury concentrations in snowpacks, 2004.

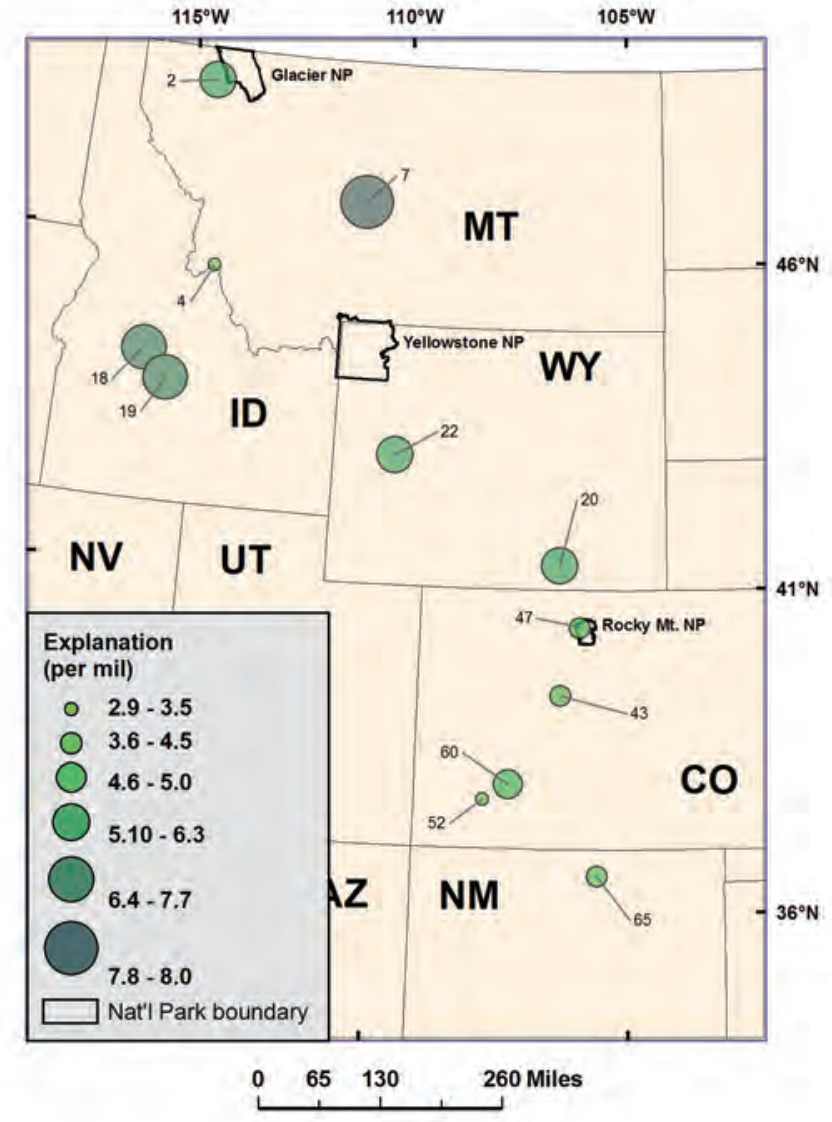

Figure 6. Relative stable sulfur isotope ratios in snowpacks, 2004. 


\section{Summary and Conclusions}

In the Rocky Mountain region, population growth, water use, and energy development are increasingly affecting the quantity and quality of water resources at higher elevations (Fenn and others, 2003; Bureau of Land Management Wyoming, 2006; McGuire, 2006; U.S. Environmental Protection Agency, 2006a, b). Identifying changes in water quality and processes leading to degradation of water quality is important because alpine and subalpine environments in the region are sensitive to changes in chemical composition of the water. Although efforts to reduce nitrogen and sulfur emissions are ongoing nationally for large point sources like powerplants (U.S. Environmental Protection Agency, 2006c), continuing growth and development likely will result in greater numbers of large and small emissions sources (such as mobile sources with gasoline and diesel engines) in the region (U.S. Environmental Protection Agency, 2006d). Atmospheric input of mercury to sensitive areas may affect aquatic and terrestrial plant and wildlife populations and has been associated with fish advisories for surface-water bodies in all States in the study area except Wyoming (U.S. Environmental Protection Agency, 2001, 2004).

Although several watershed-scale studies have investigated anthropogenic deposition in small headwater basins in the Rocky Mountain region (Turk and Campbell, 1987; Caine and Thurman, 1990; Baron, 1992; Reuss and others, 1993; Campbell and others, 1995; Williams and others, 1996; and Burns, 2002), regional-scale atmospheric deposition data are sparse. The National Atmospheric Deposition Program (NADP) provides nationwide estimates of atmospheric deposition (Nilles, 2000; National Atmospheric Deposition Program, 2004a). However, coverage for high-elevation areas (greater than 2,400 meters) in the Rocky Mountains is limited. Although 13 NADP sites monitor atmospheric deposition above 2,400 meters in Colorado, few sites are operated in the high-elevation areas of Idaho, Montana, Wyoming, and New Mexico, where snowpacks persist with negligible melt through the snowfall season. These high-elevation snowpacks are important because they may accumulate two to three times or more of the annual precipitation measured at lower elevations where regular monitoring is more feasible.

To gain a better understanding of atmospheric deposition at high elevation in the Rocky Mountains, the U.S. Geological Survey, in cooperation with the National Park Service, U.S. Department of Agriculture, Forest Service, and other organizations established a network of more than 50 snow-sampling sites in the Rocky Mountain region. Sites in the network have been sampled annually since 1993 . This report presents the 2004 snowpack chemistry data as an annual update and for comparison to data collected in previous years.

Snowpack samples were collected in a network of 65 geographically distributed sites extending from New Mexico to Montana near the end of the 2004 snowfall season. The 2004 snowfall season was drier than usual, and when snowpack samples were collected in February, March, and April, the snowpack snow-water equivalent (SWE) was near average to well below average throughout most of the Rocky Mountain region. When compared to 30-year averages for 1971-2000, SWE accumulations on April 1 were 76 to 89 percent in Montana, 64 to 81 percent in Idaho, 70 to 80 percent in Wyoming, 60 to 79 percent in Colorado, and 50 to 64 percent in New Mexico.

Snowpack-chemical concentrations yielded the following regional patterns. Ammonium concentrations in 2004 were lower than average concentrations for the 12-year period 1993-2004 at 61 percent of sites in the region. However, in northern Colorado, three of the four highest ammonium concentrations in the region occurred at sites in and adjacent to Rocky Mountain National Park. These elevated ammonium levels likely were related to emissions from highly productive agricultural areas of northeastern Colorado. Nitrate concentrations in 2004 were lower than the 12-year average for the year at 53 percent of regional sites. Lower nitrate concentrations typically occurred at sites in Wyoming, Idaho, and Montana where powerplants and large industrial areas were limited. By contrast, Colorado led the region with 9 of the 10 highest nitrate concentrations detected at sites in the State. Sulfate concentrations generally were lower in the less-developed areas of the northern one-half of the region (with the exception of the two sites in central Idaho), and higher in the southern one-half of the region. A regional reduction of sulfate concentrations across most of the Rocky Mountains was consistent with results of other monitoring of atmospheric deposition in the Western United States. Total mercury concentrations decreased slightly for the region as a whole in 2004 relative to 2003. Concentrations generally were highest in northern New Mexico, Colorado, and southern Wyoming; the highest concentration was at Brooklyn Lake, Wyoming (the highest mercury concentration in the region also occurred at this site in 2003). Lower mercury concentrations were observed from northwestern Wyoming northward into Montana, particularly in northwestern Wyoming; the lowest concentration was observed at Elkhart Park. Stable sulfur isotopic ratios had a similar regional pattern as observed in recent years with $\delta^{34} \mathrm{~S}$ values generally increasing northward from northern New Mexico to northern Colorado, Wyoming, Idaho, and Montana.

Data presented in this report reflect changing atmospheric deposition to snowpacks as concentrations across the Rocky Mountain region increase or decrease over time. Sulfate concentrations in 2004 appeared to be on the decline at 84 percent of the snowpack sites. However, ammonium and nitrate concentrations were elevated in Colorado. Although efforts to reduce nitrogen emissions are ongoing nationally, continuing growth and development likely will result in greater numbers of mobile and small-emissions sources in the region. Further, national programs to reduce emissions such as sulfur dioxide are not in place for ammonium, so increasing concentrations of ammonium in the southern one-half of the region, in particular, may be seen in the future. Continued monitoring of ammonium, nitrogen, sulfate, and mercury concentrations in Rocky Mountain snowpacks will be important as the region continues to be developed. 


\section{References Cited}

Baron, J.S., ed., 1992, Biogeochemistry of a subalpine ecosystem-Loch Vale watershed: New York, Springer-Verlag, $247 \mathrm{p}$.

Bureau of Land Management Wyoming, 2006, Pinedale Field Office, Jonah Infill Drilling Project, Draft Environmental Impact Statement, http://www.wy.blm.gov/nepa/pfodocs/ jonah/index.html, accessed October 2006.

Burns, D.A., 2002, The effects of atmospheric nitrogen deposition in the Rocky Mountains of Colorado and southern Wyoming-A synthesis and critical assessment of published results: U.S. Geological Survey Water-Resources Investigations Report 02-4066, 36 p.

Caine, Nel, and Thurman, E.M., 1990, Temporal and spatial variations in the solute content of an alpine stream, Colorado Front Range: Geomorphology, v. 4, p. 55-72.

Campbell, D.H., Clow, D.W., Ingersoll, G.P., Mast, M.A., Spahr, N.E., and Turk, J.T., 1995, Processes controlling the chemistry of two snowmelt-dominated streams in the Rocky Mountains: Water Resources Research, v. 31, p. 2811-2821.

Fenn, M.E., Haeuber, Richard, Tonnesen, G.S., Baron, J.S., Grossman-Clark, Suzanne, Hope, Diane, Jaffe, D.A., Copeland, Scott, Geiser, Linda, Rueth, H.,M., and Sickman, J.O., 2003, Nitrogen emissions, deposition, and monitoring in the Western United States: BioScience, v. 53 no. 4, p. 391-403.

Ingersoll, G.P., Turk, J.T., Mast, M.A., Clow, D.W., Campbell, D.H., and Bailey, Z.C., 2002, Rocky Mountain snowpack chemistry network - History, methods, and the importance of monitoring mountain ecosystems: U.S. Geological Survey Open-File Report 01-466, 14 p. http://water.usgs.gov/ pubs/of/2001/ofro1-466/

Ingersoll, G.P., Mast, M.A., Clow, D.W., Nanus, Leora, Campbell, D.H., and Handran, Heather, 2003, Rocky Mountain snowpack chemistry at selected sites for 2001: U.S. Geological Survey Open-File Report 03-48, 11 p. http://water. usgs.gov/pubs/of/2003/ofr0348/

Ingersoll, G.P., Mast, M.A., Nanus, Leora, Manthorne, D.,J., Clow, D.W., Handran, Heather, and Winterringer, Jesse, and Campbell, D.H., 2004, Rocky Mountain snowpack chemistry at selected sites, 2002: U.S. Geological Survey Open-File Report 2004-1027, 15 p. http://pubs.usgs.gov/ of/2004/1027/

Ingersoll, G.P., Mast, M.A., Nanus, Leora, Manthorne, D.J., Campbell, D.H., Handran, Heather, Hultstrand, D.H., and Winterringer, J., 2005, Rocky Mountain snowpack chemistry at selected sites, 2003: U.S. Geological Survey OpenFile Report 2005-1332, 15 p. Available online at: http:// water.usgs.gov/pubs/of/2005/1332/
Lehmann, C.M.B., Bowersox, V.C., and Larson, S.M., 2005, Spatial and temporal trends of precipitation chemistry in the United States, 1985-2002: Environmental Pollution, v. 135, p. $347-361$.

Mast, M.A., Turk, J.T., Ingersoll, G.P., Clow, D.W., and Kester, Cindy, 2001, Use of stable sulfur isotopes to identify sources of sulfate in Rocky Mountain snowpacks: Atmospheric Environment, v. 35, p. 3303-3313.

McGuire, Kim, 2006, "Western Oil, gas drilling will double, analysis says,": The Denver Post, October 19, 2006, http:// www.denverpost.com/news/ci_4513790, accessed October 2006.

National Atmospheric Deposition Program, 1993, NADP/NTN Annual data summary, precipitation chemistry in the United States, 1992: Fort Collins, Colorado State University, Natural Resource Ecology Laboratory, 480 p.

National Atmospheric Deposition Program. 2004a, National Atmospheric Deposition Program 2003 Annual Summary: Champaign, Illinois State Water Survey, NADP Data Report 2004-01.

National Atmospheric Deposition Program, 2004b, (NRSP-3)/ Mercury Deposition Network: Champaign, Illinois State Water Survey, NADP Program Office, http://nadp.sws.uiuc. $e d u / m d n /$, accessed July 2006.

National Atmospheric Deposition Program, 2006, Animated Isopleth Maps: http://nadp.sws.uiuc.edu/amaps2/, accessed June 2006.

National Resources Conservation Service, 2004, National Weather and Climate Center: http://www.wcc.nrcs.usda.gov/ cgibin/past_up.pl, accessed December 2004.

Nilles, M.A., 2000, Atmospheric deposition program of the U.S. Geological Survey: U.S. Geological Survey Fact Sheet 112-00, $6 \mathrm{p}$.

Reuss, J.O., Vertucci, F.A., Musselman, R.C., and Sommerfeld, R.A., 1993, Biogeochemical fluxes in the Glacier Lakes catchments: Fort Collins, Colo., U.S. Department of Agriculture, Forest Service, Rocky Mountain Forest and Range Experimental Station, General Technical Report RM-314, 27 p.

Thompson, R.S., Whitlock, C., Bartlein, P.J., Harrison, S.P., Spaulding, W.G., 1993, Climate changes in the Western United States since 18,000 yr B.P., in Wright, and others, eds., Global climates since the last glacial maximum: University of Minnesota Press, p. 469.

Turk, J.T., and Campbell, D.H., 1987, Estimates of acidification of lakes in the Mt. Zirkel Wilderness Area, Colorado: Water Resources Research, v. 23, p. 1757-1761. 
Turk, J.T., Taylor, H.E., Ingersoll, G.P., Tonnessen, K.A., Clow, D.W., Mast, M.A., Campbell, D.H., and Melack, J.M., 2001, Major-ion chemistry of the Rocky Mountain snowpack, USA: Atmospheric Environment, v. 35, p. 3957-3966.

U.S. Environmental Protection Agency, 2001, Factsheet, Mercury Update: Impact on Fish Advisories: http://www.epa. gov/ost/fishadvice/mercupd.pdf, accessed December 2004.

U.S. Environmental Protection Agency, 2004, Mercury emissions and electric utilities, February 24, 1998: http://cfpub. epa.gov/gdm/, accessed December 2004.

U.S. Environmental Protection Agency, 2006a, NOx: What is it? Where does it come from?: http://www.epa.gov/oar/ noxfldr.pdf, accessed October 2006.

U.S. Environmental Protection Agency, 2006b, SO2: What is it? Where does it come from?: http://www.epa.gov/air/ urbanair/so2/what1.html, accessed October 2006.

U.S. Environmental Protection Agency, 2006c, Acid Rain Program 2004 Progress Report: http://www.epa.gov/airmarkets/ cmprpt/arp04/2004report.pdf

U.S. Environmental Protection Agency, 2006d, National NOx Emissions Inventory: http://www.epa.gov/omswww/regs/ hd-hwy/hdria26f.pdf\#search='emissions\%20inventory\%20 NOX'

U.S. Environmental Protection Agency, 2006e, Clean Air Markets-Data and Maps: http://cfpub.epa.gov/gdm/index. cfm?fuseaction=whereyoulive.nation, accessed July, 2006.

U.S. Environmental Protection Agency, 2006f, Air Trends: Sulfur Dioxide: http://www.epa.gov/airtrends/sulfur.html, accessed July 2006.

Western Regional Climate Center, 2004, SNOTEL Maps, http://www.wrcc.dri.edu/snotel/snoareas.html, accessed July 2007.

Williams, M.W., Baron, J.S., Caine, N., Sommerfeld, R.A., and Sanford, R., Jr., 1996, Nitrogen saturation in the Rocky Mountains: Environmental Science and Technology, v. 30, p. 640-646. 